\title{
Research on undergraduate thesis's tutor allocation based on Dynamic Game Model of Incomplete Information
}

\author{
Hengwei Zhang ${ }^{1,2}$ \\ ${ }^{1}$ Zhengzhou Institute of Information Science and Technology, \\ Zhengzhou 450001, China, \\ ${ }^{2}$ State Key Laboratory of Mathematical Engineering and Advanced Computing, Zhengzhou 450001, \\ China
}

Email: wade.heat@163.com

Key words: Undergraduate Thesis; Tutor Allocation; Dynamic Game

\begin{abstract}
Fairness is the prerequisite to the undergraduate thesis's tutor allocation, which means students have the priority for choosing tutors. Because of rich experience and authoritative discourse power, the senior tutors are usually the objects that students compete for. In the case of information asymmetry, resources of the scarce senior tutors may not be fully utilized, resulting in the decline of thesis quality. In this paper we analyze the efficiency of the existing undergraduate thesis's tutor allocation by using signaling game model. Finally, the improved allocation is proposed, meanwhile some advices on prompting thesis quality are given.
\end{abstract}

\section{Introduction}

As the comprehensive practical teaching process, the writing of undergraduate thesis, through which students' abilities in mastering knowledge, analyzing problems and solving them can be examined and developed, is an important reflection of combining theory with practice ${ }^{[1]}$. At the same time, the quality of undergraduate thesis is an important basis for measuring the teaching effect, and also is the direct reflection of the quality of higher education ${ }^{[2]}$. However, the quality of undergraduate thesis recently declines significantly. Some scholars appeal to cancel the writing of undergraduate thesis. Aimed at the decline phenomenon, scholars have discussed a lot from many aspects. In [3], the authors hold the view that the main reason of the decline is formalism of school, lacking guidance of teachers and perfunctory handle of students. They pointed the key to enhance the quality of undergraduate thesis is to raise awareness of the importance of thesis writing and to make great efforts in the basic writing process. In [4], the author emphasized that due to employment pressure, bad practice effect and the lack of time and vigor, the undergraduate may not write the thesis seriously, which is the origin of the decline of thesis quality. He advised a practice mechanism should be established, by which employment can be guaranteed beforehand. Thus, students' attitude of thesis writing can be turned serious, enhancing the quality of undergraduate thesis in essence. In [5], authors suggested thesis writing should be conducted in advance. Besides, theme selection of the thesis should be objective. And the whole process should be supervised. There is no doubt that the above objectively analyzes the partial reason of the decline of thesis quality and also gives some effective advice. Nevertheless, in order to explore more factors to improve the thesis quality of undergraduate, we propose an improved tutor's allocation based on signaling game model in this paper.

\section{Fundamental assumptions and the general model}

Now we make the following fundamental assumptions for the subsequent analysis.

Assumption 1: There are two types of the undergraduates. One type indicates the students with good foundation and the right attitude to the thesis writing, who really hope to write brilliant thesis(represented by G), and the other indicates the students with poor foundation, who only want the thesis passed(represented by B). Each probability of the two types is $1 / 2$. 
Assumption 2: There are two types of the tutors. One indicates the senior tutors who are of rich experience and authoritative discourse power, and the other indicates those who lack experience. And the two types are represented by $\mathrm{S}$ and $\mathrm{J}$ respectively. The quantity of the senior tutors is less, which is in accordance with the general colleges.

Assumption 3: Types of the students are the private information. Though types of students can be reflected by their academic achievement to a certain extent, there are so many students that tutors teaching a certain course cannot classify the right students' types. On the contrary, types of tutors are the common information for the students since the quantity of tutors is relatively smaller and their information on qualification can be easily gotten.

Assumption 4: Students' payoffs of completing the thesis consist of the positive effectiveness from thesis's scores and the negative effectiveness from the cost of thesis writing. Tutors' payoffs consist of the positive effectiveness from thesis's scores and the negative effectiveness from the cost of the thesis guidance. The cost and effectiveness for tutors of different types are different in directing the students of different types, and the cost and effectiveness for students of different types are also different under the direction of the tutors of different types, because of which there is a need to make a detailed assumption, as Tab.1 and Tab. 2 shows:

\begin{tabular}{|c|c|c|c|c|}
\hline & $\begin{array}{c}\text { Thesis } \\
\text { score }\end{array}$ & $\begin{array}{c}\text { Junior } \\
\text { tutor(J) }\end{array}$ & $\begin{array}{c}\text { Senior } \\
\text { tutor(S) }\end{array}$ & $\begin{array}{c}\text { Effectiveness of } \\
\text { thesis score }\end{array}$ \\
\hline \multirow{2}{*}{$\begin{array}{c}\text { Good } \\
\text { student(G) }\end{array}$} & Passed & $C_{J, p}^{G}$ & $C_{S, p}^{G}$ & $U_{S, p}^{G}$ \\
\cline { 2 - 5 } & Brilliant & $C_{J, b}^{G}$ & $C_{S, b}^{G}$ & $U_{S, b}^{G}$ \\
\hline \multirow{2}{*}{$\begin{array}{c}\text { Bad } \\
\text { student(B) }\end{array}$} & Passed & $C_{J, p}^{B}$ & $C_{S, p}^{B}$ & $U_{S, p}^{B}$ \\
\cline { 2 - 5 } & Brilliant & $C_{J, b}^{B}$ & $C_{S, b}^{B}$ & $U_{S, b}^{B}$ \\
\hline
\end{tabular}

Tab.1. Cost and effectiveness for students of different types to complete

a thesis under the direction of tutors of different types

According to the above the assumptions, we can draw the conclusion from Tab.1: $C_{H, p}^{G}<C_{L, p}^{G}<C_{L, b}^{G}, C_{H, p}^{G}<C_{H, b}^{G}<C_{L, b}^{G}, C_{H, p}^{B}<C_{L, p}^{B}<C_{L, b}^{B}, C_{H, p}^{B}<C_{H, b}^{B}<C_{L, b}^{B}$. Besides, the cost of good students is less than the bad students'. The relationship of effectiveness of thesis scores is: $U_{S, p}^{G}<U_{S, b}^{G}, U_{S, p}^{B}=U_{S, b}^{B}$. For the bad students, they only want the thesis passed, so the brilliant thesis is equal to the rightly passed thesis.

\begin{tabular}{|c|c|c|c|c|}
\hline & $\begin{array}{c}\text { Thesis } \\
\text { score }\end{array}$ & $\begin{array}{c}\text { Good } \\
\text { student(G) }\end{array}$ & $\begin{array}{c}\text { Bad } \\
\text { student(B) }\end{array}$ & $\begin{array}{c}\text { Effectiveness of } \\
\text { thesis score }\end{array}$ \\
\hline \multirow{2}{*}{$\begin{array}{c}\text { Senior } \\
\text { tutor(S) }\end{array}$} & Passed & $C_{G, p}^{S}$ & $C_{B, p}^{S}$ & $U_{T, p}^{S}$ \\
\cline { 2 - 5 } & Brilliant & $C_{G, b}^{S}$ & $C_{B, b}^{S}$ & $U_{T, b}^{S}$ \\
\hline \multirow{2}{*}{$\begin{array}{c}\text { Junior } \\
\text { tutor(J) }\end{array}$} & Passed & $C_{G, p}^{J}$ & $C_{B, p}^{J}$ & $U_{T, p}^{J}$ \\
\cline { 2 - 5 } & Brilliant & $C_{G, b}^{J}$ & $C_{B, B}^{J}$ & $U_{T, b}^{J}$ \\
\hline
\end{tabular}

Tab. 2. Cost and effectiveness for tutors of different types to direct students of different types to complete thesis

Similarly, we can conclude from Tab. 2 based on the above assumptions: $C_{G, p}^{S}<C_{B, p}^{S}<C_{B, b}^{S}$, $C_{G, p}^{S}<C_{G, b}^{S}<C_{B, b}^{S}, C_{G, p}^{J}<C_{B, p}^{J}<C_{B, b}^{J}, C_{G, p}^{L}<C_{G, b}^{L}<C_{B, b}^{L}$. Besides, the cost of senior tutors is less than junior tutors'. The relationship of effectiveness of directing students is: $U_{T, p}^{S}<U_{T, b}^{S}$, $U_{T, p}^{J}<U_{T, b}^{J}$, and $U_{T, b}^{S}<U_{T, b}^{J}, U_{T, p}^{S}<U_{T, p}^{J}$. As for junior tutors, they may be much more delightful than senior tutors if the junior tutors can successfully direct students to complete thesis.

In order to embody the fairness of education, the undergraduate thesis's tutor allocation tends to 
respect the rights of students, which entitles the student to select tutors before the tutor's selection to students. After the selection of the student to a certain tutor, the tutor selects students according to the number of students who can be directed. Thus, we can regard the above process as a game model of information asymmetry. Students (including good students and bad students) have advantage of information can release the corresponding signals $m_{1}$ and $m_{2}$. With the above assumption, the senior tutors are the optimal selection for good students or bad students. But the numbers of students who can be directed by senior tutors and vigor of senior tutors are limited. The senior tutors must select some of those students. As the types of students are private information, it is difficult for senior tutors to select students for the maximum effectiveness. Therefore, we should design a mechanism for the undergraduate thesis's tutor allocation to make the resources of the senior tutors fully utilized.

The game model is as follows:

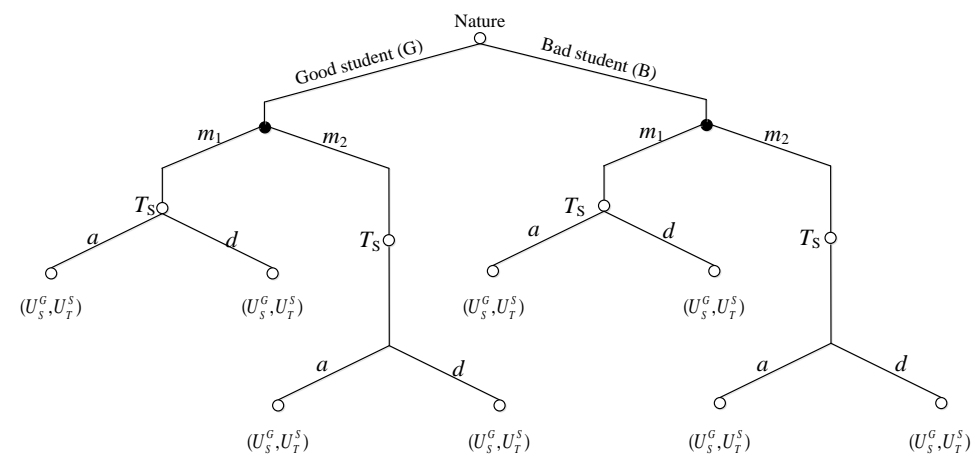

Fig.1. Signaling Game Tree

(The student selects the senior tutor.)

If separating equilibrium of the above signaling game model exists, which means students release the signals which can reflect the real types of themselves, senior tutors accept good students and decline bad students. Then the tutor allocation is efficient, which can enhance the thesis quality under given conditions.

\section{Efficiency analysis of the existing tutor allocation}

There are generally two tutor allocations for the undergraduate thesis: (1) After the selection of students to tutors, the tutors select students according to the number of students that can be directed. (2) Students select the thesis themes proposed by tutors beforehand to match the corresponding tutor, in which the same thesis theme cannot be selected by different students. Then if the number of students who select a certain tutor exceeds the limit, the tutor select several students from the all who select the tutor.

Apparently, the first allocation definitely is almost same with the above general game model. The second one seems fairer than the first, which is the completely anonymous two-way choice, meanwhile the interest of students is also considered. But it is hard to say the second one is more efficient than the first, because in the game both players of the second allocation would not make their effectiveness maximized to make decision. Further analysis shows there is no essential difference between the first allocation and the second, since in the second allocation students may only have addition cost for knowing who proposes the thesis theme through several approach. Therefore, we only choose the first allocation to analyze the existing allocation efficiency.

Under the circumstance of the existing undergraduate thesis's tutor allocation, in order to maximize his payoffs the student initially releases the signal beneficial to himself to be selected by the senior tutor. Then he shows the real type in the thesis writing and negotiates with the tutor. For instance, the bad student may ask the tutor to low the demand, since on the given condition the tutor would like to make the compromise, which accord with the demand to maximize his own payoffs. The payoff of the tutor's selection to the player in signaling game model is showed in figure2. 


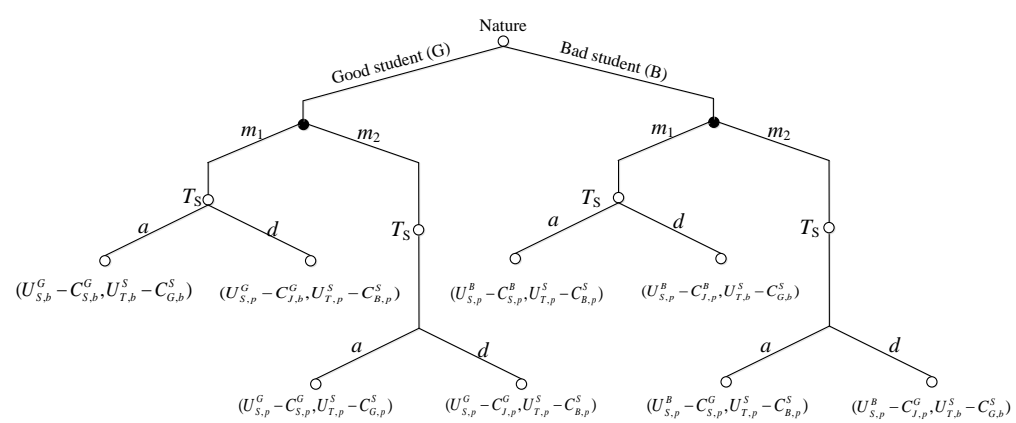

Fig.2. Signaling Game Tree

(The existing undergraduate thesis's tutor allocation)

From figure2, there are only two results of separate equilibrium: (1) When $T_{S}$ receives the signal $m_{1}$, the senior tutor $T_{S}$ looks upon the student as a good one with the strategy selection $a$. When receiving the signal $m_{2}$, the senior tutor $T_{S}$ looks upon the student as a bad one with the strategy selection $d$. (2) When $T_{S}$ receives the signal $m_{2}$, the senior tutor looks upon the student as a good one with the strategy selection $a$. When receiving the signal $m_{1}$, the senior tutor $T_{S}$ looks upon the student as a bad one with the strategy selection $d$.

Now, we should ascertain whether the above two results are the real separate equilibrium. As for the result (1), given the judgment criterion of $T_{S}$, if the good student releases the signal $m_{1}$, the senior tutor $T_{S}$ will select the strategy $a$. In this situation, the whole payoff of the good student is $U_{S, b}^{G}-C_{H, b}^{G}$, which is larger than the payoff $U_{S, p}^{G}-C_{L, p}^{G}$ gotten by releasing the signal $m_{2}$, so the signal $m_{1}$ is the optimal selection for the good student. If the bad student releases the signal $m_{1}$, the senior tutor will select the strategy a, whose payoff is $U_{S, p}^{B}-C_{H, p}^{B}$, which is larger than $U_{S, p}^{B}-C_{L, p}^{B}$ gotten by releasing the signal $m_{2}$, so the signal $m_{1}$ is the optimal selection for the bad student. The above analysis indicates the result (1) is not the real separate equilibrium, which means the good student or the bad student shows the senior tutor himself the good one.

Since the senior tutor usually would not select the one who boasts himself a good student, the result (2) is unreasonable. Similarly, we analyze the result (2) and then can conclude both the good student and the bad student would release the signal $m_{2}$ to indicate him the bad one. So the result (2) is also not the real separate equilibrium.

As the above analysis shows, the existing undergraduate thesis's tutor allocation is not really reasonable, which cannot make the players including students and tutors maximize their payoffs. Good student or bad student indicates himself the good one to the senior tutor. Once the bad student is selected by the senior tutor, he would ask the tutor to reduce requirements. Thus, the bad student maximizes his payoff, but the rare brilliant resources of the senior tutor are greatly wasted, which decline the quality of the undergraduate thesis.

\section{An improvement in the tutor allocation}

Now, we analyze the efficiency of the undergraduate thesis's tutor allocation giving the tutor the convincement that thesis writing must be brilliant. Due to the authentic convincement, the bad student must cost $C_{S, b}^{B}$ larger than previous cost to complete a piece of brilliant thesis. Meanwhile, the tutor must cost $C_{S, b}^{S}$ which is also larger than the previous one. Payoff of the tutor's selection to the players in signaling game model is showed in figure3. 


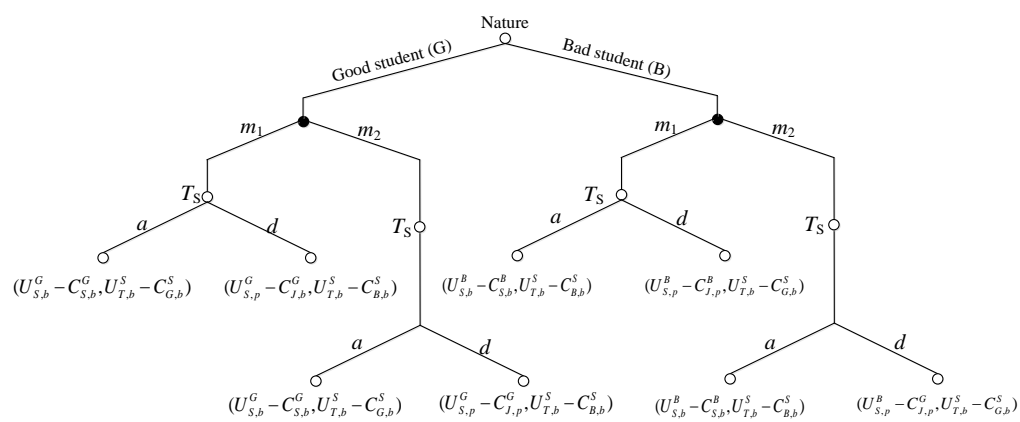

Fig.3. Signaling Game Tree

(The improved undergraduate thesis's tutor allocation)

Similarly, as figure3 shows, there are only two results of separate equilibrium: (1) When $T_{H}$ receives the signal $m_{1}$, the senior tutor $T_{S}$ looks upon the student as a good one with the strategy selection $a$. When receiving the signal $m_{2}$, the senior tutor $T_{S}$ looks upon the student as a bad one with the strategy selection $d$. (2) When $T_{S}$ receives the signal $m_{2}$, the senior tutor looks upon the student as a good one with the strategy selection $a$. When receiving the signal $m_{1}$, the senior tutor $T_{S}$ looks upon the student as a bad one with the strategy selection $d$.

Now, we should ascertain whether the above two results are the real separate equilibrium. As for the result (1), given the judgment criterion of $T_{S}$, if the good student releases the signal $m_{1}$, the senior tutor $T_{S}$ will select the strategy $a$. In this situation, the whole payoff of the good student is $U_{S, b}^{G}-C_{H, b}^{G}$, which is larger than the payoff $U_{S, p}^{G}-C_{L, p}^{G}$ gotten by releasing the signal $m_{2}$, so the signal $m_{1}$ is the optimal selection for the good student. If the bad student releases the signal $m_{1}$, the senior tutor will select the strategy a, whose payoff is $U_{S, p}^{B}-C_{H, p}^{B}$, which is smaller than $U_{S, p}^{B}-C_{L, p}^{B}$ gotten by releasing the signal $m_{2}$, so the signal $m_{2}$ is the optimal selection for the bad student. The above analysis indicates the result (1) is the real separate equilibrium, which means the good student or the bad student shows the senior tutor his real type.

For the result (2), it is also a separate equilibrium that the good student declare himself the bad one and the bad student declares himself the good one, which is scarcely existed in the real scene.

\section{Conclusion}

Through the analysis of the efficiency of the existing undergraduate thesis's tutor allocation, based on signaling game model, it shows the unreasonable results. Then we make a modification to improve the tutor allocation. Obviously, we give the senior tutor a convincement that the thesis writing must be brilliant, so separate equilibrium can be formed in the previous general signal game model. Both the good student and the bad student reflect their real type to the senior tutor, which results in the full utilization of the rare brilliant resources and can enhance the quality of the undergraduate thesis. Therefore, the improvement proposed in the paper is efficient.

\section{References}

[1] Qiao Jun, Men Qing-ling. Some thought of improving the quality of undergraduate course graduation thesis[J]. EDUCATION EXPLORATION. 2011, Serial No. 243.

[2] Xu Hua-dong, Shen Mei-hua. Thought of guiding undergraduate course graduation design[J]. Educator.2013(9):64.

[3] Wang Jian, Lan Yu-jie, Wang Shao-feng. The route choice of enhancing the quality of the university undergraduate course graduation thesis[J]. Journal of Fuyang Teachers College(Social Science). 2011, No.4. 
[4] Kang Hong-mei. The problem existing in the undergraduate course graduation thesis, causes and coping strategies[J]. CAREER HORIZON. 2014.02.

[5] Yang Ze-lin, Geng Guang-hua. Research on the current quality problems of the undergraduate course graduation thesis and efficient improved strategies[J]. Education and Vocation. 2012, (27):184-185. 